\title{
The role of the complex textural microstructure co- occurrence matrices, based on Laws' features, in the characterization and recognition of some pathological structures, from ultrasound images
}

\author{
Delia Mitrea, Sergiu Nedevschi, Mihail Abrudean, Radu Chifor and Radu Badea
}

\begin{abstract}
The non-invasive diagnosis, based on ultrasound images, is a challenge in nowadays research. We develop computerized, texture-based methods, for automatic and computer assisted diagnosis, using the information obtained from ultrasound images. In this work, we defined the cooccurrence matrix of complex textural microstructures determined by using the Laws' convolution filters and we experimented it in order to perform the characterization and recognition of some important anatomical and pathological structures, within ultrasound images. These structures were the colorectal tumors and the gingival sulcus, the properties of the latter being important concerning the diagnosis and monitoring of the periodontal disease. We determined the textural model of these structures, using the classical and the newly defined textural features. For the automatic recognition, we used powerful classifiers, such as the Multilayer Perceptron, the Support-Vector Machines, decision-trees based classifiers such as Random Forest and C4.5, respectively AdaBoost in combination with the $\mathrm{C} 4.5$ algorithm.
\end{abstract}

Keywords-Complex Textural Microstructure Co-occurrence Matrix (CTMCM), classification performance, non-invasive diagnosis, texture, ultrasound images.

\section{INTRODUCTION}

As they frequently affect the population of the developed countries and constitute a lethal disease, the colorectal tumors represent an important issue nowadays. The most reliable methods for diagnosis, the biopsy, the colonoscopy and the endoscopy are invasive or dangerous. The ultrasonography is a non-invasive method that is also inexpensive, safe, having the possibility of repeatability. In ultrasound images, the colorectal tumors have the characteristics of heterogeneity, bowel wall thickening and increased vascularity [1]. An eloquent example of a colorectal tumor is depicted in Fig. 1(a). We developed computerized, texture-based methods, for the

Manuscript received on November 22, 2015, revised February 29, 2016. Delia Mitrea and Sergiu Nedevschi are with the Technical University of ClujNapoca, Computer Science Department, Baritiu Str., No. 26-28, 400027, Cluj-Napoca, Romania, (corresponding author e-mail: Delia.Mitrea@ cs.utcluj.ro)

Mihail Abrudean is with the Technical University of Cluj-Napoca, Automation Department, Baritiu Str., No. 26-28, 400027, Cluj-Napoca, Romania.

Radu Badea is with the Medical Imaging Department, Iuliu Hatieganu University of Medicine and Pharmacy, V. Babes Str., No. 8, Cluj-Napoca, Romania.

Radu Chifor is with the Department of Preventive Dentistry, Iuliu Hatieganu University of Medicine and Pharmacy, V. Babes Str., No. 15, 400012 Cluj-Napoca, Romania. non-invasive diagnosis of the colorectal tumors, based on ultrasound images. The Inflammatory Bowel Diseases (IBD) constitute chronic affections that frequently affect the population in the modern era, consisting mainly of the bowel layer inflammation, resembling the colorectal tumors in many situations, in ultrasound images [2]. For this reason, we compared the colorectal tumors with the IBD in our work.

The periodontal disease (periodontitis) is extremely widespread in the population nowadays. Gingival bleeding is highly prevalent among adults, and advanced stages of the periodontal disease affect $10 \%$ to $15 \%$ of them [3]. The size of the gingival sulcus (the possible space between the tooth and the gum, lined by sulcular epithelium) is an important property to be assessed in order to monitor the evolution of the periodontal disease, as the gingival sulcus becomes larger with the disease evolution). In ultrasound images, the gingival sulcus has a hypoechogenic, homogeneous aspect, an eloquent example being illustrated in Fig. 1(b). The delimitation of the gingival sulcus from the neighboring regions (teeth and gingival tissue) is difficult to be performed, within ultrasound images, by the human eye or by employing usual image analysis methods. We aim to find appropriate features in order to characterize the visual aspect of the gingival sulcus within high-frequency ultrasound images, this being a preliminary step for performing an accurate segmentation of this structure.

Texture is an important property of the body tissues, able to provide subtle information concerning both the tumor structure and the gingival sulcus aspect within ultrasound images [4]. Texture-based methods, in combination with classifiers, were widely used in the scientific research nowadays [5], [6], [7], [8], [9]. In our previous research, we defined the textural model of some important affections, consisting of the most relevant textural features for their characterization and of the specific values that corresponded to the relevant textural features: arithmetic mean, standard deviation, probability distribution [10]. In this work, we defined and implemented the complex textural microstructure co-occurrence matrix (CTMCM) of order two and three. The third order CTMCM was computed at a single resolution, as well as in a multiresolution manner. We assessed the role of the CTMCM matrix upon the improvement of the textural model of the colorectal tumors, respectively in order to accurately characterize the gingival sulcus within highfrequency ultrasound images.

The paper is organized as follows: first, the state of the art 
is provided in the next section; then, we define the CTMCM matrices and we describe the feature selection and classification methods implemented in this work. We describe the experiments performed in order to assess the role of the CTMCM in the automatic diagnosis of the colorectal tumors, respectively in order to perform the characterization and recognition of the gingival sulcus within ultrasound images. At the end, we formulate the conclusions and provide the bibliographic references.

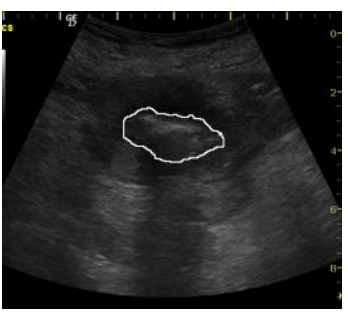

(a)
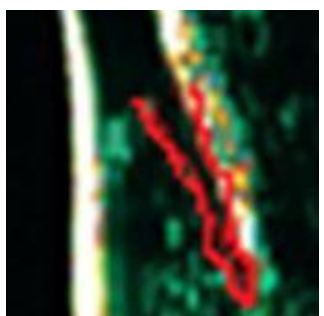

(b)
Fig.1. (a.) Colorectal tumor in ultrasound image (marked contour); (b.) Gingival sulcus in high-frequency ultrasound image (marked contour)

\section{THE STATE OF THE ART}

The most often implemented texture-analysis methods in the domain of pathological structure recognition from medical images were the second order GLCM matrix and the associated Haralick features, the Run-Length matrix [5], the fractals [6], the Wavelet [7] and Gabor transforms [8], used in combination with the k-nn classifiers, Bayesian classifiers [8], Artificial Neural Networks (ANN), Fisher Linear Discriminants, or Support Vector Machines (SVM) [5]. The resulting accuracy of these methods was around $90 \%$. Concerning the automatic diagnosis of the colorectal cancer, in [9] the authors employed the Grey Level Co-occurrence Matrix (GLCM) in combination with morphologic features referring to shape and orientation, in order to distinguish the malignant and bening tissues in the case of the patients affected by colorectal cancer. These features were computed based on biopsy slides. The maximum obtained accuracy in this case was about 90\% [9]. The generalized superior order co-occurrence matrices based on grey levels or edge orientations were defined in [10], [11]. A Local Binary Pattern (LBP) Co-occurrence Matrix of order two was defined in [12] and assessed on various datasets from the Brodatz texture collection, the resulted average accuracy being $94.30 \%$. Also, a "Texture and Texture Orientation Cooccurrence Matrix" based on the computation of edge orientations, as well as on texton detection using specific filters, was defined and experimented in [13], yielding an average accuracy of $95.5 \%$. There not exist significant approaches referring to co-occurrence matrices of the textural microstructures, determined by using the Laws' convolution filters. In [14], we defined the simple textural microstructure co-occurrence matrix, computed after the application of each Laws' filter. In this work, we study the role that the Complex Textural Microstructure Co-occurrence Matrix (CTMCM) has in the characterization and automatic diagnosis of some important structures within ultrasound images: the colorectal tumors, respectively the gingival sulcus, as it appears in the case of the periodontal disease.

\section{THE PROPOSED METHODS}

In order to assess the role of the CTMCM matrices in the recognition of the colorectal tumors, we first applied the newly defined texture analysis methods, then we performed feature selection using specific techniques and, at the end, we applied supervised classifiers in order to evaluate the classification performance. The techniques corresponding to this methodology are detailed below.

\section{A. The newly defined texture analysis methods}

The Complex Textural Microstructure Co-occurrence Matrix (CTMCM) was determined through the methodology described below, consisting of the following steps: (1) First, we associated feature vectors to the pixels in the region of interest, consisting of the results obtained after applying the 2D Laws' convolution filters for detecting levels, edges, spots, waves, ripples and also combined microstructures (L5L5, E5E5, S5S5, W5W5, R5R5, S5R5, R5S5) [15]. (2) Then, we applied an improved $k$-means clustering method, in the following manner: we started from a minimum number of centers $(k=50)$; this number was increased by splitting the corresponding centers; a center was split in two other centers, if the standard deviation of the items within the corresponding class (cluster) overpassed the threshold equal with $3 / 4$ of the average standard deviation of all the existing classes. The newly resulted centers were computed as being $1 / 2$ of the old center, respectively $3 / 2$ of the old center. (3) All the labels of the pixels from the ROI were re-assigned after splitting the old centers. The condition for the algorithm to finish was the convergence, the maximum number of centers being established to 200. The solution of the algorithm (the optimal solution) corresponded to the minimum value of WCSS (Within Cluster Sum of Squared Errors) [3]. Thus, the definition of the Complex Textural Microstructure Cooccurrence Matrix - CTMCM is provided in (1):

$$
\begin{aligned}
& C_{D}\left(t_{1}, t_{2}, . ., t_{n}\right)=\#\left\{\left(\left(x_{1}, y_{1}\right),\left(x_{2}, y_{2}\right),\left(x_{3}, y_{3}\right), . .,\left(x_{n}, y_{n}\right)\right):\right. \\
& A\left(x_{1}, y_{1}\right)=t_{1}, A\left(x_{2}, y_{2}\right)=t_{2}, . ., A\left(x_{n}, y_{n}\right)=t_{n}, \\
& \left|x_{2}-x_{1}\right|=\left|\vec{d} x_{1}\right|, x_{3}-x_{1}|=| \vec{d} x_{2}|, . .,| x_{n}-x_{1}|=| \vec{d} x_{n-1} \mid, \\
& \left|y_{2}-y_{1}\right|=\left|\vec{d} y_{1}\right|,\left|y_{3}-y_{1}\right|=\left|\vec{d} y_{2}\right|, \ldots,\left|y_{n}-y_{1}\right|=\left|\vec{d} y_{n-1}\right|, \\
& \operatorname{sgn}\left(\left(x_{2}-x_{1}\right)\left(y_{2}-y_{1}\right)\right)=\operatorname{sgn}\left(\vec{d} x_{1} \cdot \vec{d} y_{1}\right), . . \\
& \left., \operatorname{sgn}\left(\left(x_{n}-x_{1}\right)\left(y_{n}-y_{1}\right)\right)=\operatorname{sgn}\left(\vec{d} x_{n-1} \cdot \vec{d} y_{n-1}\right)\right\}
\end{aligned}
$$

In (1), \#S is the number of elements of the set $S$ and

$$
\vec{d}=\left(\left(\vec{d} x_{1}, \vec{d} y_{1}\right),\left(\vec{d} x_{2}, \vec{d} y_{2}\right), . .,\left(\vec{d} x_{n-1}, \vec{d} y_{n-1}\right)\right)
$$

are the displacement vectors. „A" stands for the attribute associated to each pixel, while $t_{1}, t_{2}, \ldots, t_{\mathrm{n}}$ are the values of the textons (cluster labels) obtained after the application of the improved $k$-means clustering algorithm. We computed the CTMCM matrix of order two and three and we determined the corresponding Haralick parameters, in a similar way as described in [10], [11]. The CTMCM matrix was determined in two situations: a) when taking into consideration all the Laws' convolution filters; b) when considering only the selected textural microstructures, corresponding to the S5S5, R5R5, S5R5 and R5S5 convolution filters, as the spot and ripple frequency provided the best results in our previous 
work [10]. We computed the CTMCM matrix of order two and three. For the CTMCM of order two, the following directions were considered: $0^{\circ}, 90^{\circ}, 180^{\circ}$, and $270^{\circ}$. For the CTMCM of order three, the current pixel was considered in the central position and together with the other two pixels they were either collinear, or formed a right angle triangle (the current pixel being in the position of the right angle). We considered the following orientations for the two displacement vectors: $\left(0^{\circ}, 180^{\circ}\right),\left(90^{\circ}, 270^{\circ}\right),\left(45^{\circ}, 225^{\circ}\right)$, $\left(135^{\circ}, 315^{\circ}\right)$ for the case of collinear pixels; $\left(0^{\circ}, 90^{\circ}\right),\left(90^{\circ}\right.$, $\left.180^{\circ}\right),\left(180^{\circ}, 270^{\circ}\right),\left(0^{\circ}, 270^{\circ}\right),\left(45^{\circ}, 135^{\circ}\right),\left(135^{\circ}, 225^{\circ}\right)$, $\left(225^{\circ}, 315^{\circ}\right),\left(45^{\circ}, 315^{\circ}\right)$, for the right angle triangle case. The displacement vectors had the absolute value 2 , in both cases. We determined the CTMCM matrices for all the considered direction combinations of the displacement vectors, the final features resulting as an average between the Haralick features of the individual matrices. We also determined the third order CTMCM matrix at multiple resolutions, obtaining the Multiresolution Complex Textural Microstructure Co-occurrence Matrix (MCTMCM) in the following manner: (1) first, we applied the Laws' convolution filters; (2) then, we applied the improved $k$-means clustering algorithm; (3) we applied the Haar Wavelet transform recursively, twice, on the image resulted at the previous step; (4) we computed the third order CTMCM matrix on each component, at two resolution levels. In this situation, we took into account all the Laws' convolution filters (at step (1)). Besides the Haralick features derived from the CTMCM matrices, we also considered, in our experiments, the following textural features: the Haralick parameters of the second and third order GLCM, respectively of the second and third order EOCM, edge and gradient based features, the autocorrelation index, fractal-based textural features (the Hurst index), the frequency of the textural microstructures, the entropy determined at two resolution levels, after applying the Wavelet transform [4].

\section{B. The selection of the relevant features}

In order to select the relevant textural features, we applied specific methods, which yielded the best results in our experiments, these being Correlation based Feature Selection (CFS) [16] combined with genetic search, which assigned a merit to each group of features with respect to the class [16] respectively the Gain Ratio Attribute Evaluation [17], combined with the Ranker method [17]. The final relevance index for each feature was obtained by performing the arithmetic mean between the individual relevance measures provided by each method.

\section{Classification performance evaluation}

For establishing the role of the CTMCM features in the context of the colorectal tumors' automatic diagnosis, respectively of the gingival sulcus characterization and recognition, we used the classifiers of Multilayer Perceptron (MLP) [18], of Support Vector Machines (SVM) [18], as well as the decision trees based algorithm Random Forest (RF) [13]. For the same purpose, we also implemented the AdaBoost meta-classifier, in conjunction with the $\mathrm{C} 4.5$ algorithm [18], this combination being well known for its performance. In order to assess the classification process, we used the recognition rate (classification accuracy), the sensitivity (TP rate), the specificity (TN rate) and the area under ROC (AUC) [18]. The strategy of cross-validation with 5 folds was adopted in this context.

\section{EXPERIMENTS AND DISCUSSIONS}

During the experiments, we used 65 cases of colorectal tumors, respectively 65 cases of Inflammatory Bowel Diseases (IBD). The corresponding images were acquired using a Logiq 7 ultrasound machine at the same settings: 5.5 $\mathrm{MHz}$ frequency, gain of 78, respectively depth of $16 \mathrm{~cm}$. Also, 50 high frequency ultrasound images representing the gingival sulcus and the surrounding tissues, belonging to 50 patients affected by periodontal disease, were taken into account for the experimental set. These images were acquired by a DermaScan C Cortex Technology ${ }^{\circledR}$, Denmark device, at a $20 \mathrm{MHz}$ frequency. The textural features were determined, after the conversion to grey-scale, within the regions of interest selected by the user inside the considered pathological structures, using our modules, implemented in Visual $\mathrm{C}++$. The methods for the selection of the relevant textural features, respectively the classifiers, were implemented using the Weka 3.6 library. Concerning the classification methods, the John's Platt Sequential Minimal Optimization Algorithm (SMO) [17] of Weka 3.6, was applied for implementing the SVM method, with a polynomial kernel, the input data being also normalized. For the classifier of Multilayer Perceptron (MLP), we considered the specific method of Weka 3.6 (MultilayerPerceptron), containing, in the single hidden layer, a number of nodes equal with $a=$ (number_of_features + number_of_classes $) / 2$, the learning rate being tuned 0.2 , respectively the momentum $\alpha$ being 0.8 . The Random Forest (RF) classification method, with 10 trees, was implemented as well. The AdaBoostM1 meta-classifier of Weka was also employed using the J48 method (the equivalent of the $\mathrm{C} 4.5$ algorithm) as a basic learner. For classification performance evaluation, crossvalidation with 5 folds was applied [17].

\section{A. The role of the CTMCM matrix in the recognition of the pathological structures}

\section{1) Colorectal tumors/IBD differentiation}

- $\quad$ The case when all the Laws'features were considered The set of the relevant textural features for the characterization of the colorectal tumors, obtained by using the methods mentioned in Chapter III, are depicted in (3). Here, we notice the presence of the second order CTMCM features homogeneity and contrast, respectively of the third order CTMCM contrast, expressing the heterogeneity of the malignant tumors relatively to the IBD case, respectively the complex structure in grey levels of the colorectal tumors.

\{GLCM_variance, Autocorrelation_index, Wavelet_Entropy3, GLCM5_Entropy, GLCM5_variance, EOCM3_Homogeneity, EOCM3_Energy, EOCM3_Entropy, EOCM3_Contrast,

EOCM3_Homogeneity, CTMCM_Homogeneity, CTMCM_Contrast, CTMCM3_Contrast $\}$

The classification performance parameters obtained in the case of the comparison between the colorectal tumors and IBD are depicted in Table I. The maximum recognition rate, of $98.33 \%$, was obtained in the case of the SVM classifier. 
We notice the increased values of the specificity (TN rate) obtained for the first three classifiers (SVM, MLP and RF).

TABLE I. THE CLASSIFICATION PERFORMANCE PARAMETERS OBTAINED IN THE CASE WHEN THE ENTIRE SET OF THE LAWS' FEATURES WAS CONSIDERED, FOR THE DIFFERENTIATION BETWEEN THE COLORECTAL TUMORS AND IBD

\begin{tabular}{lllll}
\hline \hline & $\begin{array}{l}\text { Rec. } \\
\text { Rate }\end{array}$ & $\begin{array}{l}\text { TP } \\
\text { Rate }\end{array}$ & TN Rate & AUC \\
\hline SVM & $98.33 \%$ & $96.7 \%$ & $98.5 \%$ & $98.33 \%$ \\
MLP & $96.66 \%$ & $93.3 \%$ & $98.33 \%$ & $96.66 \%$ \\
RF & $95 \%$ & $93.3 \%$ & $96.7 \%$ & $95 \%$ \\
$\begin{array}{l}\text { AdaBoost } \\
+ \text { J48 }\end{array}$ & $93.3 \%$ & $96.7 \%$ & $90 \%$ & $93.3 \%$ \\
\hline \hline
\end{tabular}

We also compared the classification accuracies obtained in the case when using only the old textural feature set with those obtained in the case when using the entire set of textural features (including the CTMCM features). As it results from the next figure (Fig. 2), in the case when considering the entire set of Laws' features, the newly resulted recognition rates overpassed the old recognition rates, in all cases.

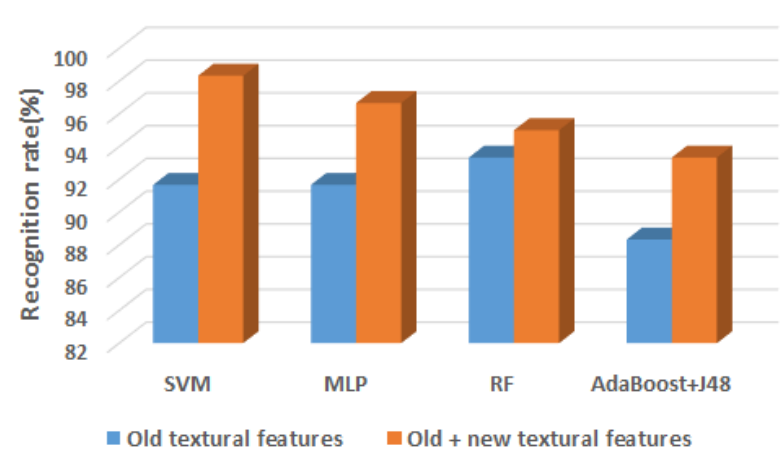

Fig. 2. The comparison between the recognition rates resulted for the old and new textural feature sets, when all the Laws' features were considered, in the case of the differentiation between colorectal tumors and IBD

- The case when only the selected Laws' features were considered

The relevant textural features obtained in this case are provided in (4). We notice the presence of the CTMCM based homogeneity, energy and contrast, as well as of the third order CTMCM based correlation and contrast, denoting the heterogeneity and complex structure of the tumoral tissue, respectively differences in granularity between the tumoral tissue and the non-tumoral one (through the third order CTMCM correlation).

\{GLCM_variance, Autocorrelation_index, Directional_grad_magnitude, Directional_grad_variance,

GLCM5_Entropy, GLCM5_Variance, EOCM3_

Homogeneity, EOCM3_Energy, EOCM3_Entropy, EOCM3_Contrast, EOCM3_Variance,

GLCM3_Homogeneity, GLCM3_Entropy,

GLCM3_Correlation, CTMCM_Homogeneity, CTMCM_Energy, CTMCM_Contrast, CTMCM3_Correlation,CTMCM3_Contrast\}

The values of the classification performance parameters obtained in this case are depicted in Table II. As it results from Table II, the maximum recognition rate obtained in this situation, of $97.5 \%$, corresponded to the RF classifier, being slightly lower than the maximum accuracy obtained in the previous case. We notice the increased values of the specificity (TN rate) in this case as well.
TABLE II. THE CLASSIFICATION PERFORMANCE PARAMETERS OBTAINED IN THE CASE WHEN THE SELECTED LAWS' FEATURES WERE CONSIDERED, FOR THE DIFFERENTIATION BETWEEN THE COLORECTAL TUMORS AND IBD

\begin{tabular}{lllll}
\hline \hline & $\begin{array}{l}\text { Recogn. } \\
\text { Rate }\end{array}$ & $\begin{array}{l}\text { TP } \\
\text { Rate }\end{array}$ & $\begin{array}{l}\text { TN } \\
\text { Rate }\end{array}$ & AUC \\
\hline SVM & $\mathbf{9 7 . 3 3 \%}$ & $\mathbf{9 4 \%}$ & $\mathbf{9 8 . 7 \%}$ & $\mathbf{9 7 . 3 \%}$ \\
MLP & $\mathbf{9 6 . 6 6 \%}$ & $\mathbf{9 1 . 7 \%}$ & $\mathbf{9 8 . 7 \%}$ & $\mathbf{9 8 . 1 \%}$ \\
RF & $\mathbf{9 7 . 5 \%}$ & $\mathbf{9 5 . 3 \%}$ & $\mathbf{9 8 . 7 \%}$ & $\mathbf{9 9 . 8 \%}$ \\
AdaBoost & $\mathbf{9 1 . 8 1 \%}$ & $\mathbf{8 6 . 3 \%}$ & $\mathbf{9 6 . 3 \%}$ & $\mathbf{9 7 . 4 \%}$ \\
+ J48 & & & & \\
\hline \hline
\end{tabular}

When performning the comparison of the entire set of the textural features (containing both the old and the newly defined features) with the old textural feature set, we noticed an increase in classification accuracy for the first mentioned set, in most of the situations. This result is illustrated in the figure below (Fig. 3).

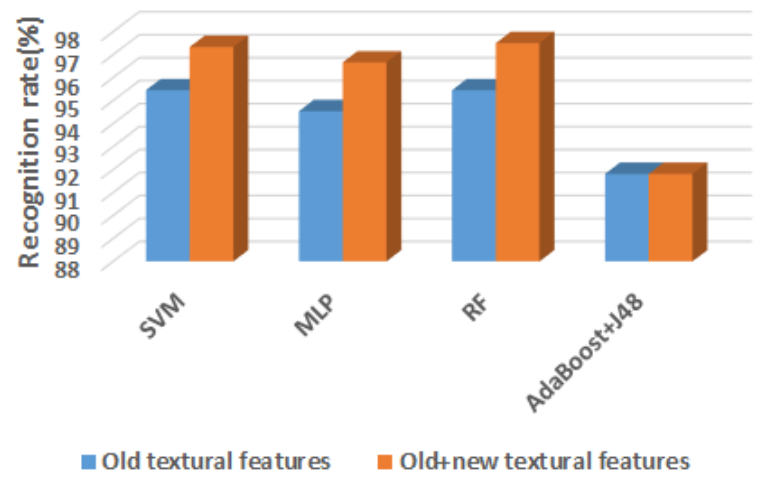

Fig. 3. The comparison between the recognition rates resulted for the old and new textural feature sets, when only the selected Laws' features were considered, in the case of differentiation between colorectal tumors and IBD

2) The differentiation between the gingival sulcus and the neighboring areas

- $\quad$ The case when all the Laws'features were considered

The set of the relevant textural features for the differentiation between the gingival sulcus and the surrounding areas is provided in (5):

\{ GLCM_Energy, Autocorrelation_index,

Edge,orientation_variability,

Wavelet_Entropy7_lh, Wavelet_Entropy7_hl,

Wavelet_Entropy7_hh, Wavelet_Entropy8_hh,

Laws_level_mean, Laws_level_frequency,

Laws_ripple_mean, Laws_ripple_frequency,

CTCM_Homogeneity, CTMCM_Entropy,

CTMCM_Correlation, CTMCM3_

Homogeneity, CTMCM3_Energy,

CTMCM3_Entropy, CTMCM3_Correlation \} \}

We notice, in (5), the presence of the second order CTMCM features, as well as of the third order CTMCM features. The homogeneity, energy and entropy, derived from the second and third order CTMCM matrices, stand for the more homogeneous and hypoechogenic nature of the gingival sulcus region, in comparison with the sourrounding areas. The correlation computed from the second, respectively from the third order CTMCM, together with the autocorrelation index, denote differences in granularity between the gingival sulcus and the neighboring regions.

The values of the classification performance parameters, obtained after the selection of the relevant textural features, in the case of separation between the gingival sulcus and the 
surrounding regions, are provided in Table III. The maximum recognition rate, of $93.05 \%$, the maximum specificity, of $91.7 \%$, as well as the maximum AUC, of $97.4 \%$, resulted in the case of the RF classifier. The maximum specificity, of $94.4 \%$, resulted in both cases of the RF and MLP classifiers.

TABLE III. THE CLASSIFICATION PERFORMANCE PARAMETERS OBTAINED WHEN ALL THE LAWS' FEATURES WERE CONSIDERED, IN THE CASE OF GINGIVAL SULCUS RECOGNITION

\begin{tabular}{lllll}
\hline \hline & $\begin{array}{l}\text { Recogn. } \\
\text { Rate }\end{array}$ & $\begin{array}{l}\text { TP } \\
\text { Rate }\end{array}$ & $\begin{array}{l}\text { TN } \\
\text { Rate }\end{array}$ & AUC \\
\hline SVM & $\mathbf{8 6 . 1 1 \%}$ & $\mathbf{8 3 . 3 \%}$ & $\mathbf{8 8 . 9 \%}$ & $\mathbf{9 4 . 7 \%}$ \\
MLP & $\mathbf{9 1 . 6 6 \%}$ & $\mathbf{9 4 . 4 \%}$ & $\mathbf{8 8 . 9 \%}$ & $\mathbf{9 2 . 1 \%}$ \\
RF & $\mathbf{9 3 . 0 5 \%}$ & $\mathbf{9 4 . 4 \%}$ & $\mathbf{9 1 . 7 \%}$ & $\mathbf{9 7 . 4 \%}$ \\
$\begin{array}{l}\text { AdaBoost } \\
+ \text { J48 }\end{array}$ & $\mathbf{8 8 . 8 8 \%}$ & $\mathbf{8 8 . 9 \%}$ & $\mathbf{8 8 . 9 \%}$ & $\mathbf{9 1 . 6 \%}$ \\
\hline \hline
\end{tabular}

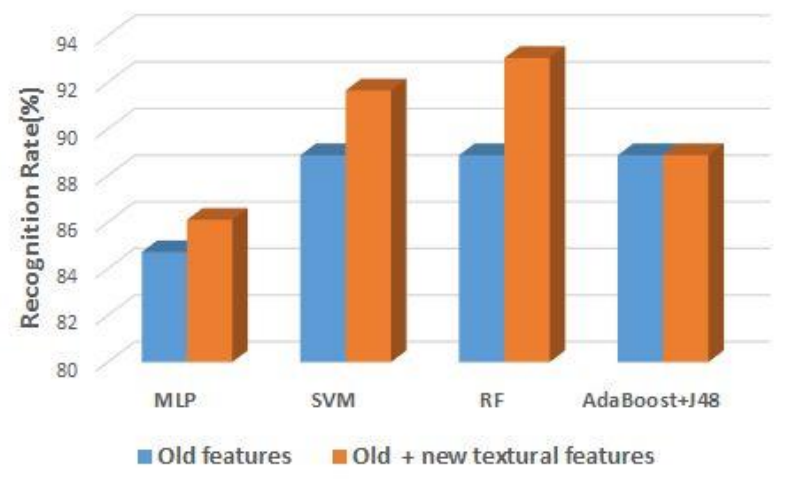

Fig. 4. The comparison between the recognition rates resulted for the old and new textural feature sets, when all the Laws' features were considered, in the case of the differentiation between the gingival sulcus and the surrounding areas

The discrimination power due to the previously existing textural features was compared with that which was due to the set formed by the old textural features and by the newly defined textural features. This comparison is illustrated in the fourth figure. From Fig. 4, we notice that the recognition rate which is due to the newly defined textural features is superior to that provided only by the old textural features in most of the situations, excepting the case of the AdaBoost metaclassifier combined with the J48 basic learner, when the two computed accuracies are equal.

- $\quad$ The case when only the selected Laws'features were considered.

The set of the relevant textural features selected in this case is illustrated in (6). We notice the presence of the textural features derived from the CTMCM matrix: the entropy computed from the second and third order CTMCM, respectively the homogeneity computed from the third order CTMCM, stand for the differences in heterogeneity between the structure of the gingival sulcus and the surrounding areas. The contrast derived from the third order CTMCM matrix denote the less complex structure of the gingival sulcus, respectively the more complex structure of the surrounding areas (gum and teeth). We also remark the first order statistics referring to the arithmetic mean and frequency of the simple textural microstructures detected by applying the Laws' convolution filters: levels, edges, spots, waves and ripples.
\{ GLCM_Energy, GLCM_Entropy, Autocorrelation_index,

Edge_orientation_variability,Wavelet_Entropy7_1l,

Wavelet_Entropy8_11,Wavelet_Entropy8_lh, Laws_level_mean, Laws_edge_mean, Laws_spot_mean, Laws_wave_mean,

Laws_ripple_mean, Laws_ripple_frequency,

CTMCM_Entropy, CTMCM3_Homogeneity, CTMCM3_Entropy, CTMCM3_Contrast \}

The classification performance parameters obtained in this situation, after the selection of the relevant textural features in the case of the differentiation between the gingival sulcus and the neighboring tissues, are illustrated in Table IV.

TABLE IV. THE CLASSIFICATION PERFORMANCE PARAMETERS OBTAINED IN THE CASE WHEN ONLY THE SELECTED LAWS' FEATURES WERE CONSIDERED,

\begin{tabular}{lllll}
\multicolumn{5}{c}{ IN THE CASE OF GINGIVAL SULCUS RECOGNITION } \\
\hline \hline & Recogn. & TP & TN & AUC \\
& Rate & Rate & Rate & \\
\hline SVM & $\mathbf{9 0 . 3 2 \%}$ & $\mathbf{9 1 . 7 \%}$ & $\mathbf{8 8 . 9 \%}$ & $\mathbf{9 7 \%}$ \\
MLP & $\mathbf{9 0 . 2 7 \%}$ & $\mathbf{9 7 . 2 \%}$ & $\mathbf{8 3 . 3 \%}$ & $\mathbf{8 8 . 7 \%}$ \\
RF & $\mathbf{9 1 . 7 5 \%}$ & $\mathbf{9 7 . 2 \%}$ & $\mathbf{8 6 . 1 \%}$ & $\mathbf{9 6 . 4 \%}$ \\
AdaBoost & $\mathbf{9 1 . 6 6 \%}$ & $\mathbf{9 4 . 4 \%}$ & $\mathbf{8 8 . 9 \%}$ & $\mathbf{8 9 \%}$ \\
$+\mathbf{J 4 8}$ & & & & \\
\hline \hline
\end{tabular}

Fig. 5 illustrates the comparison between the recognition rates obtained when considering only the old textural feature set, respectively the set formed by the old and new textural features. As it results from Fig.5, there is always an increase in accuracy for the feature set that includes the newly defined textural features. The best accuracy, of $91.75 \%$, resulted in the case of the RF classifier, being smaller than the maximum accuracy $(93.05 \%)$ resulted in the case when all the Laws' features were considered in order to define the CTMCM matrix The highest sensitivity, of $97.2 \%$ resulted in both cases of MLP and RF classifiers, the highest specificity, of $88.9 \%$ resulted in the cases of the SVM classifier, respectively of the combination between the AdaBoost metaclassifier and the J48 learner, while the highest recognition rate, of $97 \%$, resulted in the case of the SVM classifier. We notice that all the values for the recognition rate were above $90 \%$ in this situation.

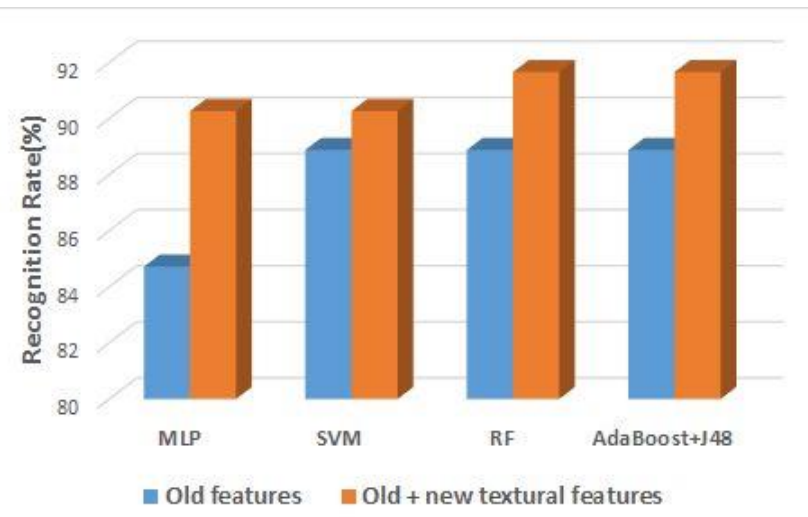

Fig. 5. The comparison between the recognition rates resulted for the old and new textural feature sets, when the selected Laws' features were considered, in the case of the differentiation between the gingival sulcus and the surrounding areas 


\section{B. The role of the multiresolution CTMCM (MCTMCM) in} the recognition of the pathlological structures

\section{1) Colorectal tumors/IBD differentiation}

The set of the relevant textural features obtained in this case is illustrated in (7). We can remark that also the third order MCTMCM based features: variance, contrast and correlation are included in this set. These features characterize the heterogeneity and the complex grey level structure of the malignant tumors. We can also notice that the relevant MCTMCM features resulted at both resolution levels: at the first level, on the component obtained by applying the combination of the low-pass filters and at the second level, on most of the components.

\{Wavelet_Entropy2, Wavelet_Entropy6_1l,

Dir_grad_variability, GLCM5_Entropy, EOCM3 Coreelation, GLCM3_Energy, MCTMCM3

_Variance2, MCTMCM3_Contrast3_ll,

MCTMCM3_Correlation2_ll, MCTMCM3 Homogeneity3_lh, MCTMCM3_Contrast3_hl, MCT MCM3_Homogeneity4_hh \}

TABLE V. THE CLASSIFICATION PERFORMANCE PARAMETERS OBTAINED IN THE CASE WHEN THE MCTMCM FEATURES WERE CONSIDERED, WHEN DIFFERENTIATING BETWEEN COLORECTAL TUMORS AND IBD

\begin{tabular}{lllll}
\hline \hline & $\begin{array}{l}\text { Recogn. } \\
\text { Rate }\end{array}$ & $\begin{array}{l}\text { TP } \\
\text { Rate }\end{array}$ & $\begin{array}{l}\text { TN } \\
\text { Rate }\end{array}$ & AUC \\
\hline SVM & $96.36 \%$ & $\mathbf{9 6 . 4 \%}$ & $\mathbf{9 6 . 4 \%}$ & $96.4 \%$ \\
MLP & $95.45 \%$ & $94.5 \%$ & $96.4 \%$ & $99.4 \%$ \\
RF & $95.45 \%$ & $92.7 \%$ & $98.2 \%$ & $98.8 \%$ \\
AdaBoost & $93.63 \%$ & $94.5 \%$ & $92.7 \%$ & $94.1 \%$ \\
+ J48 & & & & \\
\hline \hline
\end{tabular}

Concerning the classification performance resulted for the relevant feature set depicted in (7), the maximum recognition rate, of $96.36 \%$, was obtained in the case of the SVM classifier, as it results from Table V. We can also notice the increased values of the specificity obtained in this case, as well as the high AUC.

At the end, we compared the classification accuracies obtained for two datasets: for the dataset containing only the old textural features, respectively for that containing both the old and new textural features. The improvement due to the entire feature set is obvious for three classifiers, while for the RF classifier, only a slight improvement was noticed, as it results from the next figure (Fig. 6).

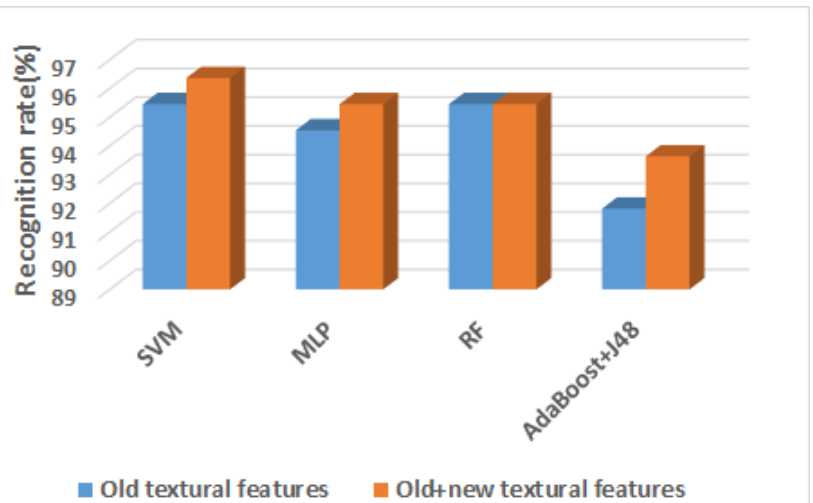

Fig. 6. The comparison between the recognition rates resulted for the old and new textural feature sets, when the MCTMCM features were considered, in the case of the Colorectal tumors/IBD differentiation
2) The differentiation between the gingival sulcus and the neighboring areas

The set of the relevant textural features for the differentiation between the gingival sulcus and the surrounding tissues, obtained when taking into account the multiresolution third order CTMCM attributes, is illustrated in (8). We notice that, in (8), there are multiple features derived from the MCTMCM matrix of order three. Thus, we remark the presence of the third order MCTMCM homogeneity, corresponding to most of the components on all the considered resolution levels, as well as of the third order MCTMCM entropy and energy, denoting the more heterogeneous structure of the tissues that surround the gingival sulcus; of the third order MCTMCM correlation, denoting differences in granularity, at multiple resolutions, between, the considered classes of tissues, of the third order MCTMCM contrast and variance, denoting the more increased structural complexity of the tissue classes that surround the gingival sulcus.

\{GLCM_Energy, GLCM_Contrast, Wavelet_Entropy7_ll, Wavelet_Entropy7_hh Wavelet_Entropy8_hh, MCTMCM3_Correlation3, MCTMCM3_Variance4, MCTMCM3_Contrast2_ll, MCTMCM3_Homogeneity4_ll, MCTMCM3_ Energy1_lh, MCTMCM3_Contrast3_lh, MCTMCM3_Variance2_lh, MCTMCM3_ Entropy2_hl, MCTMCM3_Contrast1_hl, MCTMCM3 Contrast4 hl, MCTMCM3 Homo geneity2_hl, MCTMCM3_Homogeneity3_hl, MCTMCM3_Homogeneity2 hh, MCTMCM3 Homogeneity3_hh, MCTMCM3_Homogeneity4_hh

In Table VI, the values of the classification performance parameters, obtained when providing the set of the relevant textural features illustrated in (8), at the inputs of the considered classifiers, are depicted. The maximum recognition rate, of $92.85 \%$, as well as the maximum AUC, of $96.5 \%$, resulted in the case of the RF classifier. We also notice the increased value of the sensitivity $(96.4 \%)$, obtained in the case of the MLP classifier.

TABLE VI. THE CLASSIFICATION PERFORMANCE PARAMETERS OBTAINED IN THE CASE WHEN THE MCTMCM FEATURES WERE CONSIDERED, WHEN DIFFERENTIATING BETWEEN THE GINGIVAL SULCUS AND THE NEIGHBORING

\begin{tabular}{lllll}
\multicolumn{5}{c}{ AREAS } \\
& Recogn. & TP & TN & AUC \\
& Rate & Rate & Rate & \\
\hline SVM & $\mathbf{9 0 . 2 8 \%}$ & $\mathbf{9 0 . 3 \%}$ & $\mathbf{9 0 . 3 \%}$ & $\mathbf{9 0 . 3 \%}$ \\
MLP & $\mathbf{9 1 . 0 7 \%}$ & $\mathbf{9 6 . 4 \%}$ & $\mathbf{8 5 . 7 \%}$ & $\mathbf{9 1 . 1 \%}$ \\
RF & $\mathbf{9 2 . 8 5 \%}$ & $\mathbf{9 2 . 9 \%}$ & $\mathbf{9 2 . 9 \%}$ & $\mathbf{9 6 . 5 \%}$ \\
AdaBoost & $\mathbf{9 0 . 2 8 \%}$ & $\mathbf{8 5 . 7 \%}$ & $\mathbf{9 2 . 9 \%}$ & $\mathbf{9 1 . 7 \%}$ \\
+ J48 & & & & \\
\hline \hline
\end{tabular}

The comparison between the classification accuracies resulted when considering only the old textural features, respectively when taking into account both the old and the newly defined textural features is illustrated in Fig.7. An accuracy increase for all the classifiers, corresponding to the case when also the newly defined textural features were taken into account, can be noticed in this figure. 


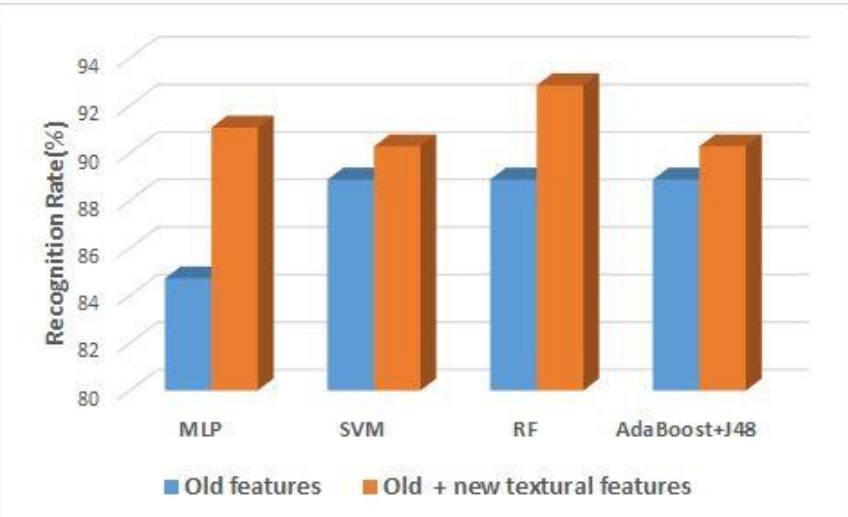

Fig.7. The comparison between the recognition rates resulted for the old and new textural feature sets, when the MCTMCM features were considered, in the case of the differentiation between the gingival sulcus and the surrounding tissues

\section{CONCLUSIONS}

The Complex Textural Microstructure Co-occurrence Matrix (CTMCM) generally led, in the case of the considered pathological structures, to a classification performance improvement, compared with the situation when using only the old textural features. The best results were obtained when all the Laws' features were considered, but satisfying results were provided also when taking into account the CTMCM matrix based only on the selected Laws' features, respectively the third order MCTMCM based features, especially in the case of the gingival sulcus characterization and recognition. The best obtained classification accuracy was $98.33 \%$ in the case of the recognition of the colo-rectal tumors and $93.05 \%$ in the case of the gingival sulcus recognition. Thus, in the first case, the result was better than the already obtained results in the domain, while in the second case the result was comparable with the state of the art results.

More extended datasets will be considered in our future work, in order to further validate the CTMCM and MCTMCM methods. Other types of multiresolution textural features will be considered as well, such as those based on the Gabor transform [4].

\section{REFERENCES}

[1] D. Martínez-Ares, I. Martín-Granizo Barrenechea, J. Souto-Ruzo, J Yáñez López, A. Pallarés Peral and J. L. Vázquez-Iglesias, "The value of abdominal ultrasound in the diagnosis of colon cancer", Revista Espanola des Enfermedades Digestiva, vol. 97, no.12, pp.877-886, 2005.

[2] L. Ruess "Inflammatory bowel disease in children and young adults: correlation of sonographic and clinical parameters during treatment", American Journal of Roentgenology, vol.175, no.1, pp.79-84, 2000.

[3] A. Nanci, D. Bosshardt, "Structure of periodontal tissues in health and disease", Periodontology 2000, vol.40, no.1, pp. 11-28, 2006

[4] A. Meyer-Base, Pattern recognition for medical imaging, Elsevier, 2009.

[5] H.Sujana, S.Swarnamani, "Application of Artificial Neural Networks for the classification of liver lesions by texture parameters", Ultrasound in Medicine \& Biology, vol. 22, no. 9, pp. 1177 -1181, 1996.

[6] T.Chikui, "Sonographic texture characterization of salivary gland tumors by fractal analysis", Ultrasound in Medicine \& Biology, vol. 31 , no. 10 , pp.1297- 1304, 2005.

[7] H.Yoshida, D.Casalino, "Wavelet packet based texture analysis for diferentiation between benign and malignant liver tumors in ultrasound images”, Physics in Medicine \& Biology, vol. 48, pp. 3735-3753, 2003.

[8] A.Madabhushi, "Automated Detection of Prostatic Adenocarcinoma from High-Resolution Ex Vivo MRI”, IEEE Transactions on Medical Imaging, pp.1611-1626, 2005.
[9] K. Masood, "Co-occurrence and morphological analysis for colon tissue biopsy classification", Proceedings of the 4th International Workshop on Information Technology, Pakistan, pp. 211-216, 2006.

[10] D. Mitrea, P. Mitrea, S. Nedevschi, R. Badea, "Abdominal tumor characterization and recognition using superior order cooccurrence matrices, based on ultrasound images", Computational and Mathematical Methods in Medicine, 17 pages, 2012.

[11] D. Mitrea, S. Nedevschi, M. Socaciu, R. Badea, "The Role of the Superior Order GLCM in the Characterization and Recognition of the Liver Tumors from Ultrasound Images", Radioengineering, vol. 21, no. 1, pp. 79-85, 2012.

[12] B. Sujatha, V. Kumar, P. Harini, "A new logical compact LBP CoOccurrence Matrix for Texture Analysis", International Journal of Scientific \& Engineering Research, vol. 3, no. 2, pp. 1-5, 2012.

[13] B. Sujatha, C. Sekhar Reddi, "Texture classification using texton cooccurrence matrix derived from texture orientation", International Journal on Soft Computing and Engineering, vol. 2, no. 6, pp. 18-23, 2013.

[14] D. Mitrea, S. Nedevschi, R. Badea, "The Role of the Textural Microstructure Coocurrence Matrices in the Classification of the Abdominal Tumors", 10th IEEE ICCP 2014 Conference, T.U.ClujNapoca, pp. 187-190, 2014.

[15] K. I. Laws, „Rapid texture identification”, SPIE Vol. 238, Image Processing for Missile Guidance, pp.76-380, 1980.

[16] M. Hall, "Benchmarking attribute selection techniques for discrete class data mining", IEEE Transactions On Knowledge and Data Engineering, vol. 15, no. 3, pp. 1-16, 2003.

[17] Weka3, Data Mining Software in Java, 2014, Online: http://www.cs.waikato.ac.nz/ml/weka.

[18] R. Duda, Pattern Classification, Wiley Interscience, 2003. 